\title{
Implicações metodológicas para o estudo do desamparo aprendido em humanos
}

\author{
Methodological implications for learned helplessness studies in \\ humans
}

Amilcar Rodrigues Fonseca Júnior, Tataína lara Moreno Pickart e Maria Cristina Zago Castelli ${ }^{1}$

[1] Centro Universitário Padre Anchieta (UNIANCHIETA), Brasil | Título abreviado: Desamparo aprendido em humanos | Endereço para correspondência: Maria Cristina Zago Castelli. Rua Bom Jesus de Pirapora, 100/140. CEP: 13207-270. Jundiaí, SP | E-mail: ajrpsico@yahoo.com.br

\begin{abstract}
Resumo: O desamparo aprendido pode ser definido como uma dificuldade de aprendizagem encontrada em sujeitos que tiveram uma história prévia com estímulos incontroláveis. No estudo desse fenômeno com humanos, alguns aspectos específicos relacionados ao controle experimental podem interferir na obtenção e na análise dos dados, entre eles: o estímulo empregado na fase de tratamento, a tarefa utilizada na fase de teste (geralmente a resolução de anagramas) e, finalmente, a condição de incontrolabilidade e as questões éticas relacionadas a ela. Estes aspectos foram discutidos criticamente no presente trabalho, considerando suas implicações na análise dos dados.
\end{abstract}

Palavras-chave: desamparo aprendido em humanos, questões metodológicas, anagramas, questões éticas

\begin{abstract}
Learned helplessness can be defined as a difficulty in learning found in subjects who had a history with uncontrollable stimuli. In the study of this phenomenon in humans, some specific aspects related to experimental control can interfere with the data acquisition and data analysis. Those aspects are: the stimulus employed in pretreatment; the task used in testing (generally anagram solution testing); and, finally, the uncontrollability condition and its ethical issues. Those aspects were critically discussed in this article, taking into account their implications for data analysis.
\end{abstract}

Keywords: learned helplessness in humans, methodological issues, anagrams, ethical issues 
O desamparo aprendido pode ser entendido como uma dificuldade de aprendizagem encontrada em sujeitos que passaram por uma história prévia com estímulos incontroláveis (Maier \& Seligman, 1976), representando um dos modelos animais que simula a depressão em humanos ${ }^{1}$ (Hiroto \& Seligman, 1975). Por meio de experimentos de laboratório, demonstrou-se que "quando um organismo sofre a experiência de um trauma que não pode controlar, sua motivação para responder quando diante de traumas posteriores se enfraquece" (Seligman, $1975 / 1977$, p. 23).

O delineamento experimental tradicional do desamparo aprendido consiste na separação dos sujeitos experimentais em três grupos, sendo eles: (a) grupo ingênuo (não tratado), que não passa pela condição experimental; (b) grupo controlável, que tem possibilidade de cessar o evento aversivo uma vez que este é apresentado; (c) grupo incontrolável, que não tem controle sobre o evento aversivo, mas é acoplado ao grupo controlável de modo que o tempo de exposição e o número de apresentações ao evento aversivo sejam iguais para ambos os grupos. Esta condição é denominada fase de tratamento.

Após esta fase, todos os sujeitos são expostos a uma nova condição, denominada fase de teste, na qual o evento aversivo é também apresentado em tentativas que duram um período de tempo determinado pelo experimentador, mas é controlável para os sujeitos dos três grupos. Nesta fase, a resposta é distinta daquela exigida na primeira fase. Assim, é possível verificar se a aprendizagem de uma nova resposta ${ }^{2}$ operante para fugir desta situação é afetada pelo tratamento anterior a que os sujeitos de cada grupo foram expostos. Geralmente, os resultados obtidos são a diminuição progressiva das latências para a resposta de fuga por parte dos sujeitos do grupo ingênuo e controlável, assim como as altas latências ou as latências máximas para a resposta de fuga por parte dos sujeitos do grupo incontrolável.

1 Para maior aprofundamento nesta questão, veja Hunziker (2005).

2 Todas as vezes que o termo resposta for mencionado será tomado como sinônimo de classe de respostas (Catania, 1998/1999).
O procedimento utilizado com humanos, com o objetivo de testar a generalidade do fenômeno do desamparo aprendido, é semelhante em praticamente todos os aspectos ao procedimento utilizado com organismos não humanos - com a ressalva de que, por questões éticas, o estímulo aversivo utilizado com humanos na fase de tratamento e na fase de teste é diferente daquele utilizado com organismos não humanos e, além disso, há o acréscimo de instruções verbais (Samelo, 2008).

Assim, nos experimentos com humanos, o controle de variáveis se mostra mais difícil. Por isso, são necessários alguns cuidados com características específicas na elaboração de delineamentos que pretendem estudar o desamparo aprendido em humanos, principalmente no que tange às características do estímulo aversivo a ser empregado na fase de tratamento, à tarefa utilizada na fase de teste e à condição de incontrolabilidade, as quais exercem papel fundamental na produção do fenômeno. Refletir sobre estes aspectos é o objetivo central deste trabalho.

\section{Fase de Tratamento: Estimulação Aversiva}

Em pesquisas com organismos não humanos existem relatos do uso de estímulos apetitivos para o estudo do desamparo aprendido (Capelari \& Hunziker, 2005; Job, 1988); porém, a generalidade desse fenômeno para contextos apetitivos ainda é fonte de divergência, de modo que não é possível afirmar, até o momento, que estes contextos são suficientes para gerar o fenômeno (Hunziker, 2005). Com humanos, a situação é similar. Assim, o uso de estimulação aversiva ainda é o mais recorrente dentro deste âmbito de estudo.

Com relação à estimulação aversiva, a apresentação de choques é comumente utilizada em organismos não humanos (Hunziker, 2005; Maier \& Seligman, 1976). No entanto, devido a restrições éticas, o leque de opções de estímulos aversivos que podem ser apresentados a humanos se mostra limitado e, portanto, é frequente na fase de tratamento a utilização de estimulação sonora (Hiroto \& Seligman, 1975; Samelo, 2008).

Segundo Perone (2003), um estímulo aversivo é definido por sua função sobre a resposta, de modo que tal função não pode ser atribuída à estrutura do estímulo, nem ser tida como inerente a ele. A função, por sua vez, depende do contexto ambiental 
no qual o estímulo é apresentado. No entanto, na fase de tratamento do desamparo aprendido, para um dos grupos os estímulos não são contingentes à resposta - o que impossibilita verificar, nesta fase, se estes estímulos são aversivos ou não com base no seu efeito sobre o responder (fuga e esquiva). Sendo assim, não há como garantir que o estímulo (estruturalmente igual) apresentado aos participantes dos grupos controlável e incontrolável exerça a mesma função aversiva nos dois contextos.

Por conta disso, o que vem sendo adotado nesta área "é que estímulos que geralmente são aversivos em contingências operantes sejam considerados aversivos mesmo se liberados de forma não contingente, desde que mantidas as suas demais características" (Hunziker \& Samelo, 2010, p. 330). Embora essa estratégia não garanta que o estímulo de fato se caracterizará como aversivo quando apresentado de forma não contingente, ela tem sido uma das mais plausíveis, visto que este problema é inerente ao procedimento desta área.

No que se refere à estimulação sonora, além da questão da aversividade do estímulo, outro cuidado deve ser tomado visando a minimizar o efeito de variáveis estranhas: selecionar adequadamente o tipo de fone de ouvido que será utilizado. A importância disso está no fato de que, a depender do modelo, alguns movimentos podem deslocar o aparelho dos ouvidos do participante e, consequentemente, interferir na intensidade do estímulo, gerando certo grau de controle. O cuidado na escolha desse item inibe variações entre participantes e torna o procedimento mais próximo daquele utilizado com animais, no qual o sujeito tem pouco ou nenhum controle sobre a intensidade do estímulo.

Com relação à fase de teste do experimento, alguns estudos utilizam anagramas como estímulos aversivos ${ }^{3}$ (Hiroto \& Seligman, 1975; Samelo, 2008). Como na fase de tratamento, nesta fase alguns cuidados também devem ser tomados, principalmente no que concerne ao uso de anagramas, como será mostrado adiante.

3 De acordo com Hunziker e Samelo (2010), em alguns casos a consequência da resolução de um anagrama pode ser positivamente reforçadora (e.g., sucesso frente ao desafio), e não negativamente reforçadora (e.g., alívio por se livrar de um problema).

\section{Fase de Teste: Resolução de Anagramas}

Hunziker e Samelo (2010) apontam que até o momento não há estímulos aversivos que se mostrem mais adequados para estudar o fenômeno do desamparo aprendido em humanos do que os anagramas. Por isso, estes estímulos vêm sendo os mais utilizados nos estudos da área. Ainda assim, as autoras enfatizam que o uso desses estímulos deve vir acompanhado de uma análise crítica.

Um anagrama é uma palavra embaralhada em uma determinada ordem ${ }^{4}$ e é considerado um equivalente tipicamente humano dos estímulos aversivos característicos da experimentação com organismos não humanos (e.g., choque), os quais controlam a aprendizagem operante. Assim, em analogia aos experimentos com organismos não humanos, seria possível observar que os participantes incluídos nos grupos ingênuo e controlável, ao tentarem solucionar os anagramas, apresentariam padrões de aprendizagem diferentes daqueles apresentados pelos participantes do grupo incontrolável. Esses últimos, por sua vez, tenderiam a apresentar dificuldades na resolução dos anagramas e, por consequência, apresentariam maiores latências.

Embora o uso dos anagramas seja comum, deve-se atentar para alguns pontos antes de escolhê-los. A repetição de uma mesma letra dentro de um anagrama pode aumentar a probabilidade de que ele seja solucionado, pois a letra repetida pode ser colocada em dois lugares diferentes, o que não é possível com as demais letras. Matematicamente, se uma letra se repete em um anagrama de cinco letras, a probabilidade de colocá-la no lugar certo, ao acaso ("chute"), em uma primeira tentativa, é de dois sobre cinco. Por outro lado, em anagramas sem letras repetidas, a probabilidade de colocar uma letra no lugar correto, ao acaso, é sempre de um sobre cinco. Assim, no anagrama NTIAT (TINTA), considerando a ordem 3-4-2-51, a mesma letra $T$ poderia ser colocada tanto no espaço da primeira letra, representada pelo número 3 na ordem apresentada, quanto no espaço da quarta letra, representada pelo número 5 nesta mesma ordem. Se qualquer outra letra for colocada em outro espaço que não o seu próprio, a palavra correta não se formará.

4 Por exemplo, considerando o anagrama STEAF - embaralhado na ordem 3-4-2-5-1-, se solucionado, formaria a palavra FESTA. 
Desta forma, quanto maior o número de letras repetidas dentro de um mesmo anagrama, teoricamente maior é a chance de acerto. Tais anagramas poderiam gerar menores latências do que aqueles que possuem todas as letras distintas umas das outras simplesmente por suas características, e não devido ao tratamento empregado ou porque o participante descobriu ou ficou sob controle da ordem em que as letras foram embaralhadas. Por se tratar de um argumento teórico, mais estudos visando a investigar o efeito de diferentes classes de anagramas sobre o responder se mostram necessários.

Geralmente, diversos anagramas são apresentados ao longo de uma mesma sessão e cada anagrama possui propriedades físicas específicas, sendo a ordem o único ponto em comum entre eles. Tal característica cria um distanciamento dos experimentos com organismos não humanos, nos quais o choque, por exemplo, apresenta quase sempre as mesmas dimensões ao longo das tentativas, como intensidade e comprimento da onda. Com isso, a ordem parece ser um importante recurso metodológico para tentar aproximar a tarefa de resolução de anagramas das tarefas tipicamente não humanas. Vale ressaltar, porém, que ter consciência da ordem não é condição necessária para que o responder fique sob controle dela.

O desconhecimento do repertório verbal dos participantes é outra questão a ser levantada em um delineamento de desamparo aprendido com humanos envolvendo anagramas. Deve-se considerar, por exemplo, que pessoas que tiveram maior contato com leitura ao longo da vida provavelmente terão um repertório mais amplo para solucionar os anagramas. Dessa forma, mostra-se útil verificar se os participantes possuem repertório básico para a realização da tarefa. Por outro lado, deve-se tomar cuidado, pois a criação de uma história pode ter efeito de imunização. Considerando esses aspectos, é possível que diferentes anagramas apresentem diferentes dificuldades e produzam diferentes resultados entre os participantes.

Por essas razões, uma classificação ${ }^{5}$ do nível de dificuldade dos anagramas a serem utilizados pode-

5 Um estudo piloto que envolva a exposição de sujeitos experimentais a situações de resolução de anagramas, com o fim de verificar as latências médias para cada anagrama, possi- ria minimizar a probabilidade futura de variações nas latências entre participantes. Recursos estatísticos seriam úteis para selecionar os anagramas que produzissem latências mais semelhantes entre si e com menores variações entre participantes. Esta ainda é uma das formas mais práticas de investigar algum tipo de homogeneidade de dificuldade existente entre os anagramas.

Uma escolha aleatória de anagramas, sem base em um sistema de classificação, poderia resultar em padrões de curvas que refletem dois efeitos conhecidos dentro do campo de estudo deste fenômeno: (a) efeito de piso, ou seja, latências baixas desde o início até o fim da fase de teste e (b) efeito de teto, isto é, latências muito altas ou latências máximas durante toda a fase de teste. O primeiro efeito poderia ser produzido pela utilização acentuada de anagramas de nível "fácil" ao longo dos blocos de tentativas, enquanto o segundo efeito poderia ser produzido pela utilização acentuada de anagramas de nível "difícil" ao longo dos blocos de tentativas, como mostra a Figura 1. Dessa forma, seria indicado que um nível "médio" pudesse ser selecionado.

Existem ainda outras duas possibilidades em que as curvas podem expressar apenas o grau de dificuldade dos anagramas, em vez do processo comportamental resultante do procedimento. Por exemplo, como mostra a Figura 2, se palavras consideradas "difíceis" se acumularem nos primeiros blocos de tentativas e palavras "fáceis" se acumularem nos últimos blocos, ter-se-ia uma curva típica de aprendizagem (a). Por outro lado, se os blocos de tentativas alternassem entre blocos com palavras "fáceis" e blocos com palavras "difíceis", obter-se-ia uma curva que expressaria "déficits de aprendizagem" (b).

De acordo com Hunziker e Samelo (2010), nesta área de estudo, a aprendizagem da tarefa, ou seja, a emissão da resposta de fuga cada vez mais rapidamente ao longo das tentativas, por parte dos participantes do grupo ingênuo, é indispensável. O comportamento desses participantes é o referente comparativo para investigar o efeito da história de incontrolabilidade sobre essa aprendizagem. Assim, supondo que os participantes dos três grupos apresentassem o mesmo padrão de cur-

bilitaria uma classificação, embora arbitrária, da dificuldade dos mesmos. 


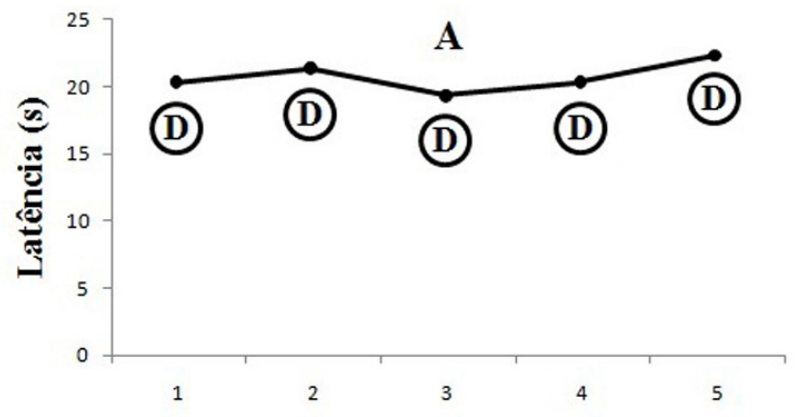

Blocos de Quatro Tentativas

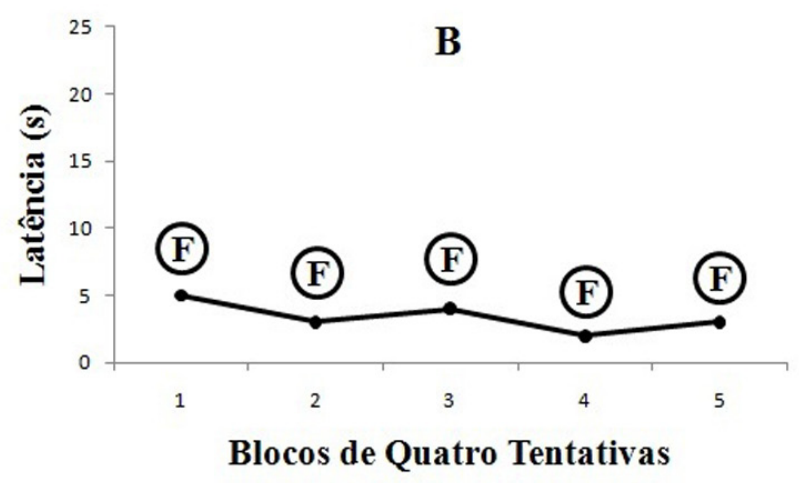

Figura 1. Gráficos hipotéticos. O Gráfico A representa um "efeito de teto" decorrente do acúmulo de anagramas de nível "difícil" (D) nos cinco blocos de tentativas. O Gráfico B representa um "efeito de piso" decorrente do acúmulo de anagramas de nível "fácil" (F) nos cinco blocos de tentativas.

va, refletindo a dificuldade dos anagramas, tanto os exemplos $A$ e $B$ da Figura 1 quanto o exemplo $B$ da Figura 2 poderiam indicar que a tarefa empregada na fase de teste não foi adequada, já que nem mesmo os participantes do grupo ingênuo aprenderam a tarefa. No entanto, se todos os participantes apresentassem o padrão de curva do exemplo $A$ da Figura 2, a fase de tratamento do experimento poderia ser questionada. Nos quatro exemplos, o efeito da variável independente seria ofuscado. Isso ressalta a importância da classificação de dificuldade dos anagramas e suscita a necessidade de uma análise cuidadosa da distribuição desses estímulos nos blocos de tentativas.

Além dessas considerações, deve-se atentar para outros fatores que podem alterar os resultados finais do experimento, ou seja, é necessário tomar alguns cuidados para que se reduza ao máximo a probabilidade de variáveis estranhas atuarem. Entre esses cuidados, vale ressaltar algumas colocações a serem feitas na instrução - se esta for utilizada -, tais como: informar o idioma das palavras, se elas estão no plural ou no singular, se possuem acentuação ou não, além da classe gramatical à qual pertencem. Por fim, desconsiderar palavras que possuem mais de uma forma de organização, como RESTO (RETOS, SETOR, SORTE), diminuiria a probabilidade de erro $^{6}$ na resolução do anagrama por um motivo que não aquele resultante do tratamento empregado.
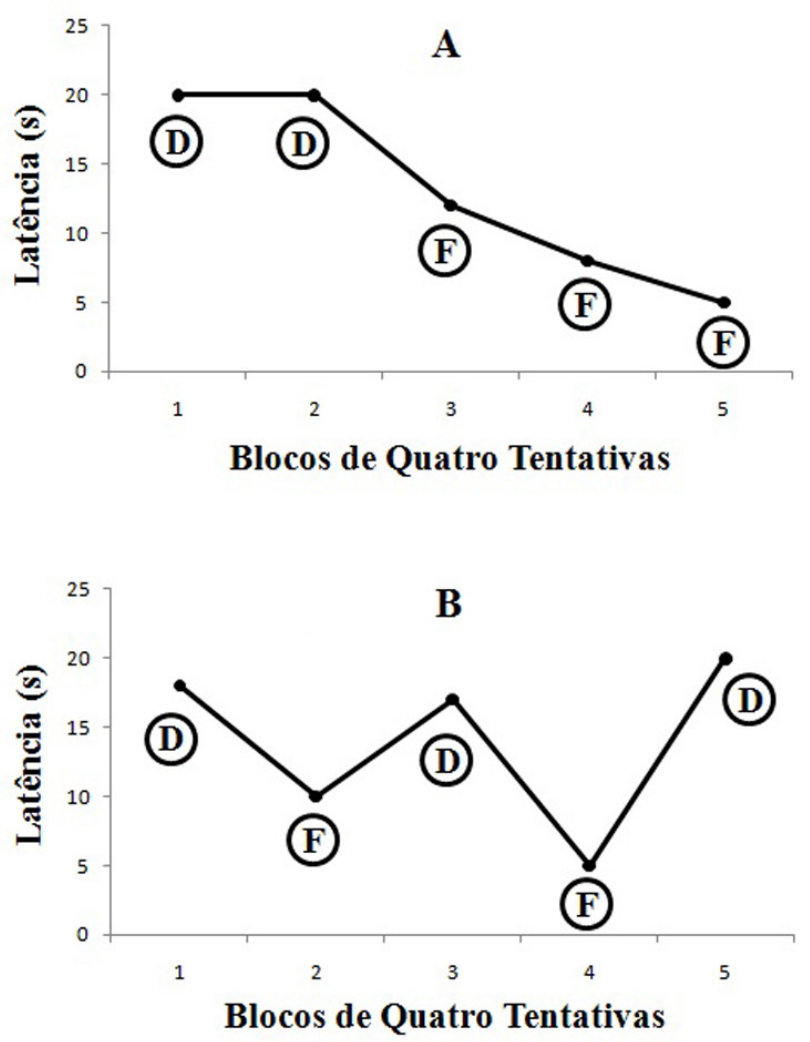

Figura 2. Gráficos hipotéticos. O Gráfico A representa uma "curva de aprendizagem" decorrente do acúmulo de anagramas "difíceis" (D) nos primeiros blocos de tentativas e de anagramas "fáceis" (F) nos últimos blocos de tentativas. O Gráfico B representa um "déficit de aprendizagem" decorrente do acúmulo alternado de anagramas "difíceis" (D) e "fáceis" (F) ao longo dos blocos de tentativas.

6 Por erro entende-se o término da latência programada pelo experimentador para a resolução do anagrama ou a formação de combinações que produzam uma palavra diferente daquela programada. 


\section{Ética}

Estresse e engodo, de acordo com Cozby (1996), são as principais questões éticas envolvidas em pesquisa. O estresse está relacionado aos danos que uma pesquisa pode causar aos participantes. Já o engodo se refere ao ato de informar os objetivos da pesquisa de forma parcial ou a atuar como um personagem para coletar dados, sem o consentimento dos participantes da pesquisa.

Devido a estas restrições éticas, a escolha do estímulo aversivo no desamparo aprendido com humanos é realizada de modo que não apresente dano de qualquer natureza aos participantes. Com relação ao engodo, porém, a questão atinge mais diretamente o rumo da pesquisa, pois afeta a variável crítica para a produção do fenômeno, ou seja, a incontrolabilidade.

Algumas informações que geralmente estão presentes no Termo de Consentimento Livre e Esclarecido (TCLE), visando a garantir a integridade dos participantes, podem fazer com que o desempenho dos mesmos, em situação experimental, seja comprometido. Por exemplo, a informação de que podem abandonar a pesquisa caso se sintam incomodados ou de alguma forma prejudicados implica certo grau de controle. Omitir tais informações, entretanto, poderia representar uma falta ética (Moraes \& Rolim, 2003). Portanto, a manutenção do TCLE se justifica, mas se caracteriza como uma das limitações deste campo de estudo com humanos.

No contexto aqui abordado, os eventos não são completamente incontroláveis, diferindo da situação experimental com sujeitos não humanos, os quais não têm a opção de abandonar a caixa experimental. Dessa forma, a diferença entre experimentos com humanos e organismos não humanos, no que concerne à metodologia, mostra-se como um fator crítico a ser levado em conta ao se comparar estas espécies e propor a generalidade do desamparo aprendido entre elas.

As reflexões aqui propostas buscaram apontar algumas das fragilidades referentes à pesquisa sobre desamparo aprendido em humanos e propor meios de lidar com algumas delas. A busca por maneiras mais refinadas de se investigar esse fenômeno se mostra válida. Por fim, dispondo-se de clareza conceitual e estando-se ciente das limitações dessa área, um maior rigor metodológico poderá ser alcançado e procedimentos mais precisos poderão ser delineados.

\section{Referências}

Capelari, A., \& Hunziker, M. H. L. (2005). Aprendizagem de fuga após estímulos apetitivos incontroláveis. Psicologia: Teoria e Pesquisa, 21, 99-107.

Catania, A. C. (1999). Aprendizagem: Comportamento, linguagem e cognição (4a ed.; D. G. Souza et al., Trads.). Porto Alegre: Artmed. (Trabalho original publicado em 1998) Cozby, P. C. (1996). Methods in behavioral research (6a ed.). California: Mayfield.

Hiroto, D. S., \& Seligman, M. E. P. (1975). Generality of learned helplessness in man. Journal of Personality and Social Psychology, 31(2), 311-327.

Hunziker, M. H. L. (2005). O desamparo aprendido revisitado: Estudos com animais. Psicologia: Teoria e Pesquisa, 21(2), 131-139.

Hunziker, M. H., \& Samelo, M. J. (2010). Muitos problemas e algumas soluções no estudo do desamparo aprendido com humanos. Em M. M. C. Hübner, M. R. Garcia, P. R. Abreu, E. N. P. Cillo, \& P. B. Faleiros (Orgs.), Sobre comportamento e cognição: Análise experimental do comportamento, cultura, questões conceituais e filosóficas (Vol. 25, pp. 328-336). Santo André: ESETec.

Job, R. F. S. (1988). Interference and facilitation produced by noncontigent reinforcement in appetitive situation. Animal Learning and Behavior, 16, 451-460.

Maier, S. F., \& Seligman, M. E. P. (1976). Learned helplessness: Theory and evidence. Journal of Experimental Psychology: General, 105, 3-46.

Moraes, A. B. A., \& Rolim, G. S. (2003). A ética em pesquisa com seres humanos: Dos documentos aos comportamentos. Em M. Z. S. Brandão, F. C. S. Conte, F. S. Brandão, Y. K. Ingberman, C. B. Moura, V. M. Silva, \& S. M. Oliane (Orgs.), Sobre comportamento e cognição: Clínica, pesquisa e aplicação (Vol. 12, pp. 353-362). Santo André: ESETec. 
Perone, M. (2003). Negative effects of positive reinforcement. The Behavior Analyst, 26, 1-14.

Samelo, M. J. (2008). Investigação sobre o desamparo aprendido em humanos (Dissertação de mestrado). Universidade de São Paulo, São Paulo, SP.

Seligman, M. E. P. (1977). Desamparo: Sobre depressão, desenvolvimento e morte (1a ed.; M. T. A. Silva \& S. M. Carvalho, Trads.). São Paulo: HUCITEC. (Trabalho original publicado em 1975)

\section{Informações do artigo}

\section{História do artigo}

Data de submissão em: 17/07/2010

Primeira decisão editorial em: 09/03/2011

Aceito para publicação em: 10/03/2011 